\title{
Editorial
}

\section{La epidemia de cesáreas como limitante del parto humanizado}

\author{
Cristian Javier Hernández-Espinosa*
}

\begin{abstract}
*Estudiante de IX semestre. Escuela de Medicina. Facultad de Salud. Universidad Industrial de Santander. Bucaramanga. Santander. Colombia. Correspondencia: Sr. Cristian Javier Hernández Espinosa. Dirección: Diagonal 35 \#197a 35, Floridablanca. Teléfono: +57 3003362794. Correo electónico: crijaheres@gmail.com
\end{abstract}

¿Cómo citar este artículo?: Hernández-Espinosa C. La epidemia de cesáreas como limitante del parto humanizado. MÉD.UIS.2019;32(1):9-12. doi: 10.18273/revmed.v32n1-2019001

\section{Parto y parto humanizado}

El parto es el proceso fisiológico mediante el cual una mujer expulsa al feto, las membranas corioamnióticas y la placenta al final de la gestación. En 1996, la Organización Mundial de la Salud (OMS) definió el parto normal como aquel que tiene un comienzo espontáneo y sin complicaciones, que cursó con un embarazo de bajo riesgo, con un niño que nace en posición cefálica entre las 37 a 42 semanas de edad gestacional, y que después de dar a luz tanto la madre como el hijo se encuentran en buenas condiciones generales ${ }^{1}$. Anualmente, ocurren más de 140 millones de nacimientos en el mundo, la mayoría sin complicaciones materno-fetales ${ }^{2}$, pero con sólo un pequeño porcentaje dentro del contexto de la normalidad, debido a la amplia gama de intervenciones que pueden realizarse durante el trabajo de parto.

Por otra parte, el parto humanizado se define como aquel que, ocurriendo en condiciones normales o no, tiene en cuenta las opiniones y necesidades de la mujer y su familia durante el parto y puerperio, de modo que se convierte en una experiencia especial y placentera donde la dignidad humana materna prevalece sobre cualquier decisión no contemplada en la medicina basada en la evidencia3. Su importancia se ha recalcado mediante la directriz de la OMS, emitida en el año 2018, que contiene 56 recomendaciones para atender el trabajo de parto.
Dicho documento propone un modelo de cuidados en las gestantes que involucra diferentes aspectos para garantizar el bienestar holístico materno-fetal (Ver figura 1) ${ }^{4}$.

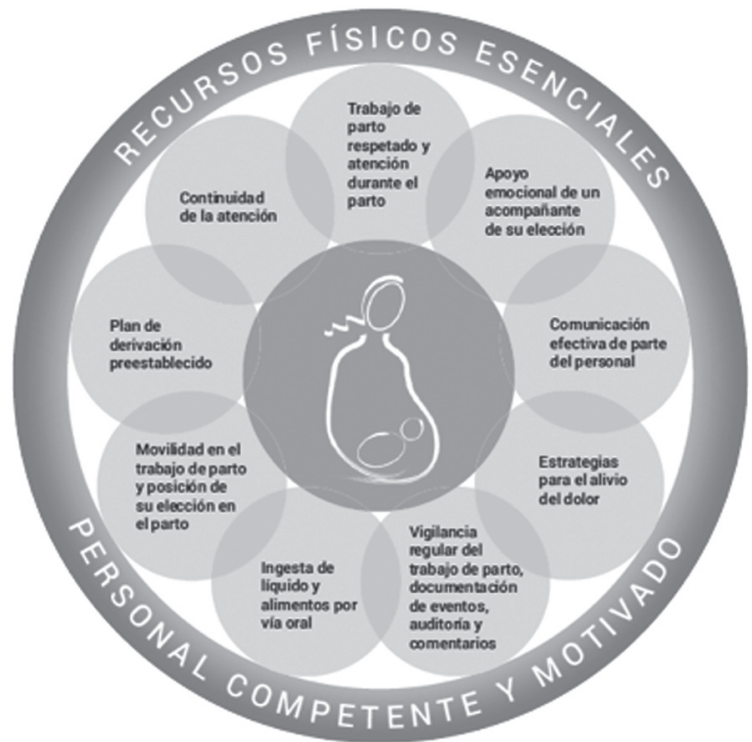

Figura 1. Esquema del modelo de cuidados durante el parto de la OMS.

Fuente: OMS. Recomendaciones de la OMS Para los cuidados durante el parto, para una experiencia de parto positiva. 2018.

Dentro de las recomendaciones, la OMS menciona aspectos que incluyen: la atención basada en el respeto, la comunicación efectiva, el acompañamiento permanente y la continuidad de la atención, la no realización de intervenciones 
rutinarias injustificadas (pelvimetría clínica al ingreso, rasurado púbico, enema al ingreso, uso de oxitocina, amniotomía, episiotomía), el respeto por los tiempos de cada período del trabajo de parto, la intervención del dolor cuando la madre lo solicite, la libertad de posición en el momento del parto, los cuidados posparto y las primeras medidas favorables al neonato (contacto piel a piel y lactancia tan rápido como sea posible $)^{4}$.

Debido a que estas recomendaciones son inherentes al trabajo de parto, y éste corresponde al proceso mediante el cual se da a luz por vía vaginal, la cesárea no es un punto que cobre relevancia dentro de la directriz propuesta por la OMS. Sin embargo, la cesárea cobra significado en el contexto del parto humanizado en la medida que se analizan las cifras relacionadas a ella y su abuso comparado con el uso necesario que sugiere la OMS.

\section{Problemática actual con la cesárea}

Si bien la cesárea es un procedimiento quirúrgico que contribuye a mejorar la morbimortalidad en mujeres con embarazos de alto riesgo que requieren dicha intervención, su sobreuso irracional corresponde a un problema que tiene implicaciones negativas sobre la ejecución del parto humanizado. Se estima que la proporción de embarazos que necesita cesárea por motivos médicos es solo del 10-15\%, pero, para el año 2018 en el 60\% de 169 países estudiados por la OMS y Unicef se encuentran cifras mayores a esta 5 .

En ese mismo año Latinoamérica se consolidó como la región con mayor proporción de cesáreas con 44,3\% de los nacimientos, siendo República Dominicana el país líder, no sólo latinoamericano, sino también mundial con 58,1\%; Brasil el país líder sudamericano con $55,5 \%$, y Colombia el cuarto país en Sudamérica con $45,9 \%$ de porcentaje de nacidos vivos por cesárea. En Santander, dicha proporción para el año 2013 fue de 53,7\%, ubicándose por encima del promedio nacional como el noveno departamento más prevalente en Colombia ${ }^{6}$. Debido a esta problemática, el uso indiscriminado de cesáreas se considera una epidemia, que no solo tiene una alta prevalencia, sino que creció a un gran ritmo en los últimos años duplicándose de $12 \%$ en el 2000 a $21 \%$ en $2015^{5}$, y que constituye una de las principales amenazas en el momento de brindar una atención humanizada para el nacimiento de los bebés.
Cesárea en mujeres con antecedente de cesárea y cesárea en primer embarazo

\section{Cesárea con antecedente de cesárea}

El incremento en la tasa de cesáreas se atribuye a varias causas; sin embargo, una de las más importantes es no intentar parto vía vaginal después de haber tenido una cesárea ${ }^{7}$. Esto significa que, de intentarse el parto vaginal después de una cesárea, se constituye una forma clave de reducir la creciente y alta tasa de las mismas. Hasta el $60-80 \%$ de las mujeres con antecedente de cesárea sometidas a Prueba de Trabajo de Parto (PTP) son exitosas ${ }^{8}$, y por ello es fundamental conocer las condiciones, en simultáneo, con las cuales es posible llevar a cabo un parto vaginal después de una cesárea (Ver tabla 1).

Tabla 1. Condiciones para llevar a prueba de trabajo de parto si existe antecedente de cesárea. Tomado y adaptado de: GarcíaBenítez CQ, López-Rioja MJ, Monzalbo-Núñez DE. Parto después de cesárea ¿una opción segura? Ginecol Obstet Mex. 2015. 83:69-87.

\begin{tabular}{|l|}
\hline \multicolumn{1}{|c|}{ Condiciones } \\
\hline Período intergenésico mayor de 18 meses. \\
Previa incisión transversal baja. \\
Único antecedente de cesárea. \\
Embarazo único. \\
Presentación cefálica. \\
Adecuado peso fetal. \\
Ninguna otra complicación. \\
\hline
\end{tabular}

Fuente: Autores.

\section{Cesárea en primer embarazo}

Otra forma muy efectiva para reducir las complicaciones que conlleva una cesárea es evitando la realización de la primera cesárea ${ }^{9}$ Dicho procedimiento incluye indicaciones obstétricas, fetales y maternas (Ver tabla 2) $)^{9,10}$.

Otras indicaciones que podrían conducir a la primera cesárea, además de las presentadas en el cuadro, incluyen: antecedente de distocia de hombros, antecedente de miomectomía, antecedente de desgarro de $3-4^{\circ}$ grado, placenta marginal y de baja implantación, obesidad, infecciones (VHS, VIH, VHC), enfermedad cardiovascular y pelvis inadecuada?

En cualquiera de las condiciones anteriormente mencionadas, la decisión final la tiene el profesional a 
Enero - Abril

cargo junto con la madre, de acuerdo con el contexto en que se presente individualizando a cada paciente y considerando riesgos y beneficios.

Tabla 2. Indicaciones mayores para realizar cesárea por primera vez. FCF: frecuencia cardíaca fetal. Tomado y adaptado de: Spong CY Berghella V, Wenstrom KD, Mercer BM, Saade GR. Preventing the first cesarean delivery: summary of a joint Eunice Kennedy Shriver National Institute of Child Health and Human Development, Society for Maternal-Fetal Medicine, and American College of Obstetricians and Gynecologists Workshop. Obstet Gynecol. 2012 Nov; 120(5):1181-93.

\begin{tabular}{|l|c|}
\hline \multicolumn{1}{|c|}{ Indicación de cesárea por primera vez } & $\%$ \\
\hline Detención de la primera etapa & $15-30$ \\
\hline Detención de la segunda etapa & $10-25$ \\
\hline Mal presentación & $10-15$ \\
\hline Inducción fallida & 10 \\
\hline FCF no tranquilizante & 10 \\
\hline Gestación múltiple & 3 \\
\hline Macrosomía & 3 \\
\hline Desórdenes hipertensivos & 3 \\
\hline Solicitud materna & $2-8$ \\
\hline
\end{tabular}

Fuente: Autores.

Sin embargo, no siempre se podrá evitar realizar la cesárea. Deben tenerse en cuenta las indicaciones que son absolutas, dentro de las cuales se encuentran: placenta previa total, vasa previa, o prolapso de cordón', en estos casos es necesario practicarla, debido a que el beneficio tanto de la madre como el hijo es mayor en comparación con el parto vaginal9.

\section{Complicaciones}

Como en toda intervención médica, la realización de la cesárea por primera vez, después de una cesárea, o la prueba de trabajo de parto implican riesgos. Aquí es donde radica la afectación del parto humanizado, debido a que muchas veces por las razones ya expuestas se conduce a la materna a realizar un procedimiento sin una indicación clara del todo, exponiéndola a complicaciones no justificadas.

Se ha descrito que la mortalidad materna en mujeres con embarazo a término es mayor si se vuelve a realizar una cesárea (9,6 por 100 000) en lugar de optar por la PTP (1,9 por 100 000) (RR 0,27 con IC 95\% 0,13-0,88) pretérmino se observa una tendencia similar con tasas de mortalidad más alta en ambos $\operatorname{casos}^{7}$. La necesidad de histerectomía también se ve reducida cuando se somete a PTP (0,22\% vs 0,43\%) (RR 0,40
La epidemia de cesáreas como limitante del parto humanizado con IC 95\% 0,18-0,92)7. Otra complicación para la cual la PTP es factor protector es la fiebre post parto, con mayor incidencia de endometritis pero sin exposición a la infección de la herida, (6,5\% vs 7,2\%) (RR 0,63 con IC $95 \% 0,43-2,76)^{7}$. Finalmente en cuanto a la estancia hospitalaria, es menor cuando se realiza PTP $(2,55$ días) en comparación con repetir cesárea (3,92 días).

En contraste, el riesgo de ruptura uterina se ve sustancialmente incrementado cuando después de una cesárea, se somete a una gestante a PTP (0,47\%) (RR 20,74 IC 95\% 9,77-44,02) en comparación con repetir cesárea (0,026\%), y este riesgo es mayor cuando se induce el trabajo de parto (2$6 \%)^{7}$. Asimismo, existe mayor riesgo de mortalidad perinatal en el mismo contexto $(1,3 \mathrm{~s} 0,5$ por 1000 nacidos) (RR 1,82 IC 95\% 1,24-2,76)7.

Sin embargo, las complicaciones no sólo afectan a la mujer durante y después del parto, sino que pueden también afectar al neonato y a los futuros embarazos maternos.

Diferente a las maternas, los neonatos generalmente presentan mayores riesgos si después de una cesárea sus madres son sometidas a PTP, debido a que en diferentes estudios se han encontrado hallazgos con los que se establecen asociaciones con encefalopatía hipóxica-isquémica, trauma secundario al trabajo de parto y distrés respiratorio neonatal; pero dentro de estos hallazgos faltan resultados en los que se compare con las primigestantes que tienen su parto por vía vaginal, ya que también son riesgos propios de este suceso ${ }^{7}$, así como otros sesgos ${ }^{11}$.

Por su parte, para la mujer en sus futuros embarazos pueden existir complicaciones implicadas en la mayor incidencia de placenta previa y el espectro placenta ácreta, que aumentan su riesgo de manera directamente proporcional con el número de cesáreas previamente realizadas`.

\section{Conclusiones}

El parto es un procedimiento que, llevado en condiciones ideales, conduce al nacimiento de un nuevo individuo con el mejor bienestar posible para el binomio madre-hijo. El hecho de realizar acciones que afecten el curso normal del parto, incluyendo la realización de cesáreas sin indicaciones justificadas de manera adecuada, representa una vulneración en el proceso humanizado del parto. Pese a que la cesárea es un procedimiento valioso, bajo ciertas 
circunstancias representa peligro tanto para la madre como para el feto, su uso debe ser ejecutado racionalmente y reducido significativamente, de modo que se eviten las complicaciones derivadas de la misma y se garantice el respeto por la dignidad humana materna.

\section{Referencias bibliográficas}

1. Organización Mundial de la Salud. Cuidados en el parto normal: una guía práctica. Departamento de Investigación y Salud Reproductiva. Ginebra. 1996.

2. SALUD. En partos, más tiempo y menos presión, pide la OMS [Internet]. Colombia: EL TIEMPO; 15 de febrero de 2018 [Consultado el lunes 25 de febrero de 2019]. Disponible en: https://www.eltiempo.com/vida/salud/oms-plantea-nuevasrecomendaciones-para-la-atencion-de-partos-183328.

3. Almaguer JA, García HJ, Vargas V. Nacimiento humanizado. Aportes de la atención intercultural a las mujeres en el embarazo, parto y puerperio. Género y salud en cifras. 2012;10(2):44-59.

4. Organización Mundial de la Salud. Recomendaciones de la OMS Para los cuidados durante el parto, para una experiencia de parto positiva. [Internet]. 2018 [Consultado el lunes 25 de febrero de 2019] Disponible en: https://apps.who.int/iris/bitstream/ handle/10665/272435/WHO-RHR-18.12-spa.pdf
5. AFP. Colombia, entre los países donde más se practican cesáreas en el mundo [Internet]. Colombia: EL TIEMPO; 12 de octubre de 2018 [Consultado el lunes 25 de febrero de 2019]. Disponible en: https://www.eltiempo.com/vida/salud/colombia-entre-los-paisesdel-mundo-que-mas-cesareas-practica-280788

6. Ministerio de salud y protección social, MINSALUD. Atlas de Variaciones Geográficas en Salud de Colombia 2015- Estudio piloto - Resultados de partos por cesárea [Internet]. Bogotá; Estudio 2015, Datos de 2013. [Consultado el miércoles 27 de febrero de 2019]. Disponible en: https://www.minsalud.gov. co/sites/rid/Lists/BibliotecaDigital/RIDE/DE/PES/Resultadosgenerales-atlas-salud-cesareas-2015.pdf

7. Sargent J, Caughey AB. Vaginal Birth After Cesarean Trends: Which Way Is the Pendulum Swinging? Obstet Gynecol Clin North Am. 2017; 44(4):655-66.

8. García-Benítez CQ, López-Rioja MJ, Monzalbo-Núñez DE. Parto después de cesárea ¿una opción segura? Ginecol Obstet Mex. 2015; 83(2):69-87.

9. Spong CY, Berghella V, Wenstrom KD, Mercer BM, Saade GR. Preventing the first cesarean delivery: summary of a joint Eunice Kennedy Shriver National Institute of Child Health and Human Development, Society for Maternal-Fetal Medicine, and American College of Obstetricians and Gynecologists Workshop. Obstet Gynecol. 2012; 120(5):1181-93.

10. Caughey AB. Evidence-Based Labor and Delivery Management, Can We Safely Reduce the Cesarean Rate? Obstet Gynecol Clin North Am. 2017;44(4):523-33.

11. Guise JM, Denman MA, Emeis C, Marshall N, Walker, Fu R, et al. New Insights on Maternal and Neonatal. Obstet Gynecol. 2010;115(6):1267-78. 